\title{
Thermostability of interface structure metal on graphene and silicene
}

\author{
E.D. Kurbanova ${ }^{1, \dagger}$, V.A. Polukhin ${ }^{1,2}$, I.V. Chepkasov ${ }^{3}$ \\ †kurbellya@mail.ru

\begin{abstract}
${ }^{1}$ Institute of Metallurgy Ural Branch Russian Academy of Sciences, Amundsen St. 101, 620016 Yekaterinburg, Russia ${ }^{2}$ Institute of Material Studies and Metallurgy, Ural Federal University, Mira St. 28, 620002 Yekaterinburg, Russia ${ }^{3}$ Khakas State University, Lenina St. 90, 655017 Abakan
\end{abstract}

Basing on molecular dynamics method the study of thermic evolution of $2 \mathrm{D}$ and $3 \mathrm{D}$ nanoclusters has been carried out. There has been revealed nature of generation and activation kinetic processes, initiated structure transformation and boosing the thermostability of functional unities of two dimentional transition metallic systems deposited on graphene and silicene substrates. Thus there has been formed by chemical bonds specific interfaces as functional unified low-dimentional systems. In the presented article results of the molecular and dynamic modeling executed with use of multiparticle potentials. There has been carried out the analysis the thermoactivity of processes of a relaxation, diffusion and formation of the interface structures metal / graphene (G) metal / silicene (Me: Ag, Ni, Al), their destruction, as analog of melting in low-dimensional systems. The diffusion components in the $X-Y$ interface planes and the normal along axis $Z$ for all considered Me/G systems depended differently on the heating temperature, reflecting the different natures of variations in the electron structure (adhesion energy and the types of sorption at interfaces). For interfaces with physical adsorption, variations in the diffusion activity differed in their smoothness with a notable rise for the contacts in the region of $\sim 1000 \mathrm{~K}$, and for $\mathrm{Al} / \mathrm{G} / \mathrm{Al}$ (with a double coating) in the region of $\sim 1800 \mathrm{~K}$, but with a different degree of nonmonotonicity. For the chemisorption interfaces formed during the hybridization of $\pi_{z}-d_{z}$ orbitals, however, slow growth at the initial stages of heating in the high-temperature region $(\sim 2000 \mathrm{~K})$ changed with an abrupt increase in both diffusion components.

Keywords: MD simulation, interface, graphene, silicene, transition metals, diffusion, thermostability.

\section{Термическая стабильность интерфейсной структуры металлов с графеном и силиценом}

\author{
Курбанова Э.Д.,, , Полухин В.А. ${ }^{1,2}$, Чепкасов И.В. ${ }^{3}$ \\ †kurbellya@mail.ru \\ ${ }^{1}$ Институт металлургии УрО РАН, ул. Амундсена 101, 620016 Екатеринбург \\ ${ }^{2}$ Институт материаловедения и металлургии УрФУ, ул. Мира 28, 620002 Екатеринбург \\ ${ }^{3}$ Хакасский государственный университет, пр. Ленина 90, 655017 Абакан
}

На основе компьютерного моделирования термической эволюции 2D и 3D нанокластеров металлов выявлены особенности зарождения и активации кинетических процессов, определяющих температуры начала структурных трансформаций и потери термической устойчивости функциональных элементов, происходящих в двумерных системах металлических нанокластеров и пленок переходных металлов, размещенных на графеновых и силиценовых подложках и образующих с ними в результате контакта специфическую поверхность раздела (интерфейс) с разными по величине энергиями связи. В представленной статье обобщены результаты молекулярно-динамического моделирования, выполненного с применением многочастичных потенциалов, проведен анализ термоактивированных процессов релаксации, диффузии и формирования интерфейсных структур металл / графен (G) и металл / силицен (Me: $\mathrm{Ag}, \mathrm{Ni}, \mathrm{Al})$, их разупорядочения и разрушении, как аналога плавления в низкоразмерных системах. Для всех рассмотренных систем $\mathrm{Me} / \mathrm{G}$ рассчитанные по среднеквадратичным смещениям компоненты диффузии в интерфейсных плоскостях $X-Y$ и нормали вдоль оси $Z$ по-разному зависели от температуры нагрева, отражая разный характер изменения электронной структуры, естественно, энергии адгезии и типа сорбции в интерфейсах. Для интерфейсов с физической адсорбцией изменение диффузионной активности отличалось плавностью с заметным ростом в области 1000 K, a для Al/G/Al (с двойным покрытием) в области $\sim 1800 \mathrm{~K}$, но разной степенью немонотонности. В то же время для хемосорбционных интерфейсов, сформированных при гибридизации $\pi_{z}-d_{z}-$ орбиталей, медленный рост на начальных этапах нагрева уже в области высоких температур ( 2000 К) менялся резким увеличением обеих компонент коэффициентов диффузии.

Ключевые слова: МД-моделирование, интерфейс, графен, силицен, переходные металлы, диффузия, термостабильность. 


\section{1. Введение}

Важнейшей проблемой при разработке функциональных материалов - сверхрпроводников остается устойчивость их слоистой структуры. Поэтому в предлагаемом исследовании на основе молекулярной динамики изучена термостабильность интерфейсных систем армированных графеном и силиценом пленок $d$-металлов, $\mathrm{Ag}, \mathrm{Al}$.

В работе на основе результатов проведенного компьютерного моделирования [1], данных газофазного синтеза [2-5] и электронной микроскопии [6] выполнен сравнительный анализ термической эволюции и стабильности интерфейсных систем $\mathrm{C}_{\mathrm{N}} / \mathrm{Me}, \mathrm{Si}_{\mathrm{N}} / \mathrm{Me}$, как структурных единиц гетероструктурно-слоистых композитов, а также течения активированных атомной динамикой процессов формирования на металлических поверхностях раздела элементарных $2 \mathrm{D}$-кластеров $\left(\mathrm{C}_{\mathrm{N}}\right.$, $\mathrm{Si}_{\mathrm{N}}$ ) в зависимости от способов их получения, характера связи и влияния природы низкоразмерных Ме подложек и нагрева. Компьютерная модель представляла собой графеновую плоскость из 300 атомов углерода с размещенными на ней металлическими пленками с исходными периодическими структурами. Нагрев проводился в интервале от $300 \mathrm{~K}$ до $3300 \mathrm{~K}$.

В ходе молекулярно-динамического моделирования взаимодействие атомов металла и атомов подложки для систем металл - углерод описаны с помощью комбинированных потенциалов по методу Рафия-Табара на основе функций Морзе с параметрами, оцененными в работе [7]. Атомов углерода или кремния - координационным потенциалами Терсофа [8]. Межатомные связи в металлах описывались потенциалом, полученным в рамках модифицированной модели ЕАM (embedded atom method), Сатона-Чена.

\section{2. Результаты и обсуждения}

Армирующие пленки силицена на металлических плоскостях высокой чистоты синтезированы методом "сухой” эпитаксии с формированием стабильной структуры поверхностей раздела при сверхвысоком вакууме [9]. Моделируемая структура поверхности раздела $\mathrm{Si} / \mathrm{Me}$ оказалась не совсем планарной (при суммарной толщине интерфейса Si-Me 1.6 нм), а ребристой (гофрированной или «волнистой»). В случае силицена эффект волнистости был не так сильно выражен $s p^{2} / s p^{3}$ гибридизованными координациями, как в случае модельных ребристых Si-сфероидов [10,11] (аналогов фуллерена $[10,11])$. Интерфейсы с кластерами на подложках Ag: $\mathrm{C}_{\mathrm{N}} /(111)-\mathrm{Ag}$ и $\mathrm{Si}_{\mathrm{N}} /(111)-\mathrm{Ag}$ отличаются распределением зарядовой плотности в зоне непосредственного контакта с поверхностью (111) - Ag [1]. Имеется сравнительно небольшое перераспределение заряда в интерфейсе $\mathrm{C} /(111)-\mathrm{Ag}$, вызванное дипольнодисперсной поляризацией как основным вкладом в силы Ван-дерВаальса (более слабых, чем силы связи при хемосорбции) и наблюдаемое лишь в контактах C-Ag по границам $\mathrm{C}_{\mathrm{N}}$-конфигураций. Согласно нашим расчетам $2 \mathrm{D}$ (планарные) конфигурации нанокластеров $\mathrm{Si}_{\mathrm{N}}$ на по- верхности (111) - Ag оказались более стабильными чем $3 \mathrm{D}$-полиэдры. B той же степени и транспорт заряда в силицене реализовывался током безмассовых высокоскоростных фермионов $\left(\sim 10^{5}-10^{6} \mathrm{~m} / \mathrm{c}\right)$ [13] (учитывая линейную зависимость дисперсии энергии $E(k)$ и равенство нулю ее второй производной).

Устойчивость координаций гофрированной структуры силицена обеспечивается гибридизацией связей $s p^{2} / s p^{3}$ и силами сорбционного типа не только по границам 2D-кристаллических фрагментов, размещенных на поверхности (111) - Ag, но и контактами $\mathrm{Si}-\mathrm{Ag}$ в центральных частях фрагментов. Так, для кластеров $\mathrm{Si}_{\mathrm{N}}$ на поверхности (111) - $\mathrm{Ag}$ энергия взаимодействия в интерфейсе растет с размерами кластеров от $E_{S} \sim 0.25$ эВ при $N=6$ до $E_{s} \sim 0.52$ эВ при $N=24[15,16]$. Другими словами эта энергия является энергией адсорбции, которая определяется в расчете на один атом $\mathrm{Si}$ (для 6 атомов 0.04 эВ/атом $\mathrm{Si}$; для 24 атомов 0.02 эВ/атом $\mathrm{Si}$ ).

Характер интерфейсных взаимодействий сказывается и на специфике температурной зависимости диффузионных движений атомов $\mathrm{Ag}$ в интерфейсах графена и силицена, что иллюстрируют графики соответствующих компонент - горизонтальной $D_{x y}$ (в плоскости $X-Y$, рис. 1(a)) и нормальной $D_{z}$ (вдоль оси $Z$ ), рис. 1(b) [14].

Таким образом, влияние силицена на динамику атомов в пленке $\mathrm{Ag}_{\mathrm{N}}$ весьма ощутимо, что следует не только из сравнительного анализа температурной зависимости коэффициентов диффузии атомов $2 \mathrm{D}$-кластеров $\mathrm{Ag}_{\mathrm{N}}$ на слоях графена и силицена, но и из изменения формы ребристости поверхности последнего за счет пассивации и эффекта параметрической несоразмерности взаимоприлегающих решеток.

Для понимания механизмов формирования рингкластеров, определяющих тот или иной тип суперрешеток силицена, и найти соответствие с экспериментально найденными структурами целесообразно также провести анализ разновидностей суперячеек, периодическая трансляция которых приводит к наименьшим эффектам параметрической несоразмерности с поверхностью (111) - Ag субстрата. Среди таковых подходящими представляются интерфейсные суперструктуры: (I) - (3×3)-Si на поверхности (111)-Ag периодичности $(4 \times 4)$ [в иной записи $(111)-\operatorname{Ag}(1 \times 1)-$ силицен];
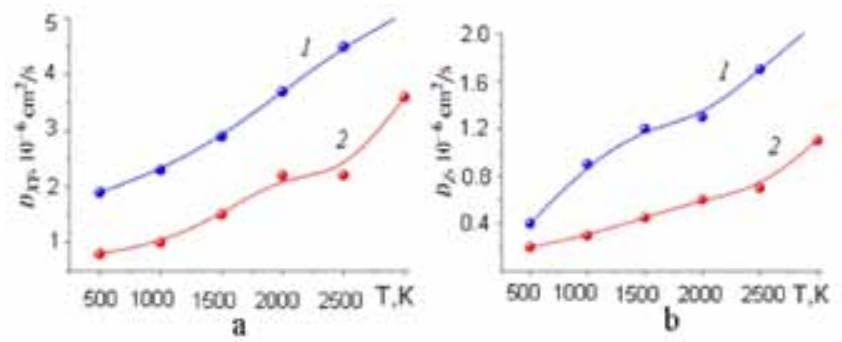

Рис. 1. Температурные изменения компонент $D_{x y}$ (a) и $D_{z}$ (b) коэффициентов диффузии атомов $\mathrm{Ag}$ c одностороннем покрытием графена (1) и силицена (2) моноатомной пленкой серебра.

Fig. 1. Temperature variations in diffusivities $D_{x y}$ (a) and $D_{z}$ (b) of $A g$ atoms with one-sided arrangement monatomic $\mathrm{Ag}$ film on graphene (1) and silicene (2). 
(II) $-\left(7^{1 / 2} \times 7^{1 / 2}\right)-\mathrm{Si} /(111)-\mathrm{Ag}$ периодичность $\left(2 \times 3^{1 / 2} \times{ }^{2} \times 3^{1 / 2}\right)$; (III) - $\left(7^{1 / 2} \times 7^{1 / 2}\right) /(111)-\mathrm{Ag} /$ периодичности $\left(13^{1 / 2} \times 13^{1 / 2}\right)[7,15]$. Анализ внешней поверхности силицена моделируемой структуры (I), рис. 2(a), (b), показал, что двойные кольца (внутренние - из 6 атомов, внешние - из 12) сформированы только из $1 / 3$ атомов $\mathrm{Si}$, что вполне согласуется с СТМ-проекциями [6]. Тогда как для структуры (II) в формировании ячеек суперрешетки участвуют только пары атомов $\mathrm{Si}$, дистанцированные на 0.576 нм и являющиеся 1/7 частью всех атомов $\mathrm{Si}$, находящихся на его внешней стороне. Из анализа суперструктуры (III), рис. 2(c), (d), следует, что в формировании гексагональных колец задействовано по одному внешнему атому Si. Таким образом, в ряду структур (I-III) уменьшается число атомов $\mathrm{Si}$, нарушающих планарность силицена, что соответствует экспериментальным наблюдениям СТМ [6], где на одну ромбическую ячейку размером 1.04 нм приходилось по одному светлому рефлексу (относящемуся к внешним атомам). Однако величина отклонения от планарности вместе с тем заметно повышается от 0.085 нм для структуры (I), до 0.119 нм для структуры (II) и до 0.139 нм для структуры (III), т. е. морфология интерфейса со структурой (I) более близка таковой интерфейсам $\mathrm{Me} / \mathrm{G}$. Таким образом, три типа смоделированных атомных структур силицена (I - III) [4], соответствующих координациям решеток, подобно полученной (I) вакуумным осаждением атомов Si на поверхность 111-Ag [16] имеют отклонение от поверхности (111)-Ag в пределах $\Delta Z$ ( 0.2 нм). Расстояние $\Delta Z$ характеризует отколонение атомов $\mathrm{Si}$ от планарности, соответствующим масштабу гибридных связей $s p^{2} / s p^{3}$, определяющих ребристость 2D-кристалла силицена. С повышением температуры и увеличением масштаба волнистого монослоя Si на поверхности (111)-Ag, согласно расчетам [15] квантовой динамики, именно орбитали $s p^{2}$ стаби- a

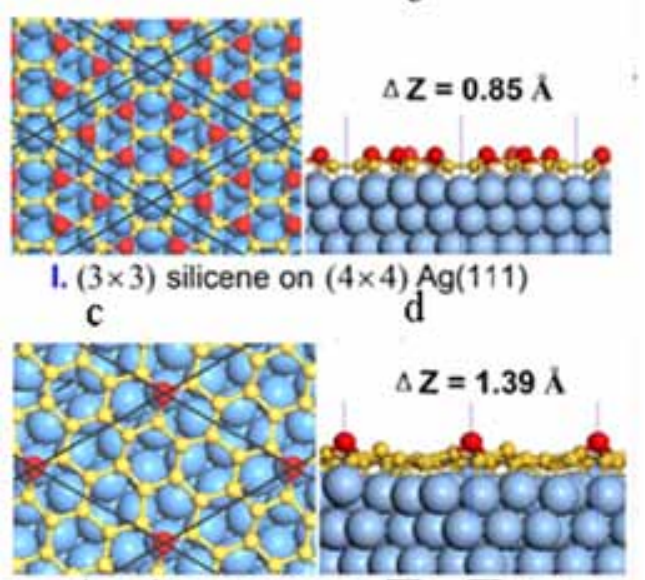

III. $(\sqrt{7} \times \sqrt{7})$ silicene on $(\sqrt{13} \times \sqrt{13}) \mathrm{Ag}(111)$
Pис. 2. Атомные структуры (I и III типа) силицена на суперструктурах серебра (a), (c) - вид сверху; (b), (d) вид сбоку; размеры максимально удаленных атомов даны с увеличением и окрашены красным.

Fig. 2. Atomic stricture (I and III types) of silicone on silver superstructure (a), (c) - top view; (b), (d) - side view; sizes are most distant atoms with the increase and painted red. лизируют подобные координации силицена. Адсорбция на поверхности (111)-Ag оказалась решающим фактором из-за гибридизации связей $s p^{2} / s p^{3}$ для достижения стабильности планарных конфигураций $\mathrm{Si}_{\mathrm{N}}$. Таким образом, при всей морфологической схожести структур поверхностей раздела $\mathrm{Ag} / \mathrm{Si}$ и $\mathrm{Ag} / \mathrm{C}$ при их формировании в механизмах сорбции имелись различия, поскольку $\mathrm{sp}^{2}$ связи углерода образовали адсорбционный монослой гексагональных координаций из атомов $\mathrm{C}$, удерживаемых у поверхности Ag диполь-дисперсионными силами между атомами С и Ag.

Сравнительный анализ термической устойчивости интерфейсов хорошо отражает температурная зависимость вертикальных (по оси $Z$ ) компонент атомной диффузии атомов Ag в соответствующих интерфейсах $\mathrm{Ag} / \mathrm{Si}$ и $\mathrm{Ag} / \mathrm{C}$, рис. 1(a), (b), соответствующие кривые 1 и 2. Конечно, в обоих случаях интенсивность диффузионной подвижности была ниже чем в пленках $\mathrm{Al}$, рис. 3(a), (b), но несравненно выше чем в интерфейсах с односторонним и двустороннем покрытием графена пленками $\mathrm{Ni}$, рис. 3(c), (d), где природа контактного взаимодействия имела хемосорбционный механизм за счет $\pi_{z}-d_{z}$ гибридизации орбиталей, соответственно, С и Ni $[1,3]$.

Присутствие кластеров $\mathrm{Al}$ (как и $\mathrm{Ni}$ ) на обеих сторонах $\mathrm{G}$ снижает в 4 раза миграцию атомов $\mathrm{C}$ и увеличивает межатомные расстояния за счет включения взаимодействия атомов металла через графеновый лист. Для кластеров $\mathrm{Al}[19], \mathrm{Ni}[9]$ и $\mathrm{Cu}[13,20]$ при однои двухстороннем их размещении на листах G более близким оказывается поведение коэффициента самодиффузии вертикальных направлений $D_{z}$. Для интерфейса $\mathrm{Al} / \mathrm{G} \mathrm{c}$ наименьшей энергией когезии металл - графен при одностороннем покрытии разупорядочение координаций атомов $\mathrm{Al}$ и термоактивируемая самосборка их в виде плоской капли наблюдались в интервале
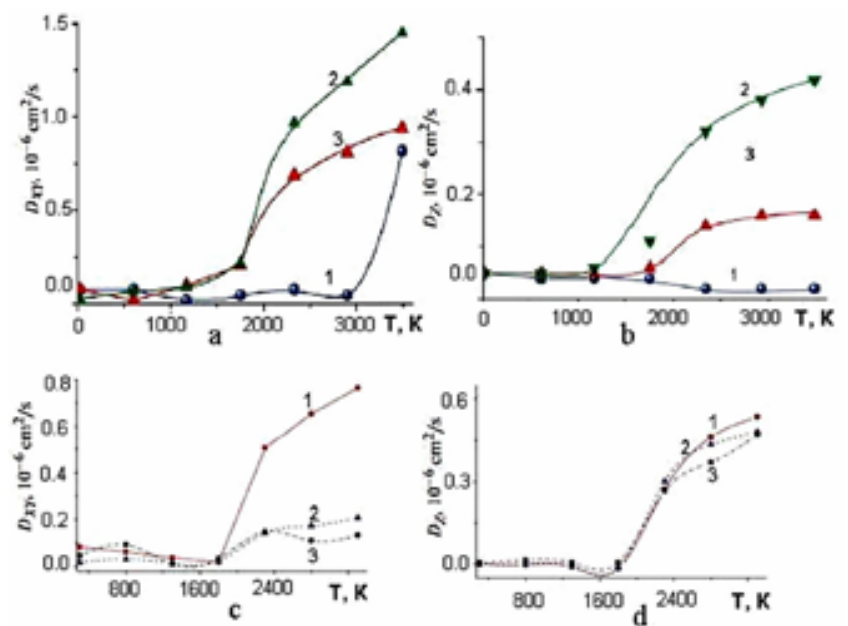

Рис. 3. Температурные изменения коэффициентов диффузии $D_{x y}$ и $D_{z}$ атомов металлов в интерфейсах: (a), (b) - Al (1 - G/Al; $\mathrm{Al} / \mathrm{G} / \mathrm{Al}$ - 2 - верхняя пленка, 3 - нижняя пленка); (c), (d) $\mathrm{Ni}(1$ - G/Ni; Ni/G/Ni - 2 - верхняя пленка, 3 - нижняя пленка.

Fig. 3. Temperature variations in diffusivities $D_{x y}$ and $D_{z}$ of metal atoms in interfaces: (a), (b) - $\mathrm{Al}(1-\mathrm{G} / \mathrm{Al} ; \mathrm{Al} / \mathrm{G} / \mathrm{Al}-2-$ upper film, 3 - bottom film); (c), (d) - Ni $(1-\mathrm{G} / \mathrm{Ni}$; Ni/G/Ni $-2-$ upper film, 3 - bottom film). 
2550 - $2650 \mathrm{~K}$, и атомы Al, несмотря на испарение, удерживались вплоть до 3250-3350 К. Тогда как при двухстороннем покрытии графена пленками эти процессы “предплавления и плавления" Al фиксировались уже в области $1300 \mathrm{~K}$ на одной из сторон графена, а процесс кипения зафиксирован в интервале $1750-1850$ К с испарением всех атомов $\mathrm{Al}$ [19]. Таким образом, термостабильность интерфейсов $\mathrm{Al} / \mathrm{G} 1$ и $\mathrm{Al} / \mathrm{G} 2$ оказалась гораздо выше, чем при таком же механизме сорбции - физической адсорбции, у интерфейса $\mathrm{Cu} / \mathrm{G}$, где исходная плотноупакованная структура размещенного на графене монослоя атомов меди претерпела полное разрушение уже свыше 300 К. Для интерфейсов с физической адсорбцией изменение диффузионной активности отличалось плавностью с заметным ростом для $\mathrm{Cu} / \mathrm{G}$ в области $\sim 1000 \mathrm{~K}$, а для $\mathrm{Al} / \mathrm{G} / \mathrm{Al}$ (с двойным покрытием) в области $\sim 1800 \mathrm{~K}$, но разной степенью немонотонности. В то же время для интерфейсов $\mathrm{Ni} / \mathrm{G}$, сформированных при гибридизации $\pi_{z}-d_{z}$-орбиталей, медленный рост на начальных этапах нагрева уже в области высоких температур ( 2000 К) менялся на резкое увеличение обеих компонент коэффициентов диффузии [21 - 23].

\section{3. Заключение}

Для всех рассмотренных интерфейсов достижимы режимы высокотемпературного разупорядочения 2D-кластеров и металлических пленок с актами термоактивированного диффузионного проникновения атомов металла на обратную сторону нанолиста G c «адгезией» их на этой стороне как в виде отдельных атомов, так и в координационно-разупорядоченных группировок.

На основе данных компьютерного моделирования проведен сравнительный анализ физико-химических условий стабилизации процессов зародышеобразования элементарных планарных 2D-кластеров $\mathrm{Si}$ и С и формирования функциональных плоскостей на субстратах (111)-Ag с подавлением ребристой дефектности структуры. Таким образом устойчивость роста 2D-кристалла силицена на (111)-Ag субстрата обусловлена $p-d$ гибридизацией орбиталей атомов из взаимоприлегающих плоскостей (111)-Ag и Si (внутренних ребристых поверхностей). Вторым немаловажным фактором является пассивация подложкой $\mathrm{Ag}$ ненасыщенных связей кремния и прежде всего периферийных (пограничных) атомов растущих нанокластеров. Оба эти фактора нивелируют дестабилизирующее влияние с одной стороны эффектов параметрической несоразмерности рингкластерной (ребристой) суперструктуры силицена и решетки Ag, с другой - это неравноценность участия самих атомов кремния в процессах сорбции как результат $s p^{2} / s p^{3}$-гибридизации с разделением на два подслоя атомов, создающих ребристость, и лежащих в основной плоскости силицена (более близких атомов к (111)-Ag поверхности). Однако с повышением температур и ростом амплитуд атомных колебаний это дестабилизирующее влияние усиливается, вызывая рост локальных напряжений в узлах рингкластерной суперструктуры силицена, что конечно будет снижать надежность материала не смотря на все его функциональные преимущества в сравнении с традиционными.

Благодарность/Aknowledgements. Работа выполнена при финансовой поддержке бюджетной научной программы ИМЕТ УрО РАН и при финансовой поддержке Правительства Российской федерации: постановление № 211, контракт № 02.A03.21.0006.

\section{Литература/References}

1. V.A. Polukhin, N.A. Vatolin. Rus. Chem. Rev. 84, 5 (2015).

2. L. Meng, Q. Sun Q, J. Wang, F.J. Ding. J. Phys. Chem. 116 (2012).

3. A. Fleurence, R. Friedlein, T. Ozaki, H. Kawai, Y. Wang, Y. Yamada-Takamura. Phys. Rev. Lett. 2 (2012).

4. I.V. Chepkasov, Yu Ya Gafner, S.L. Gafner and S.P. Bardakhanov. Bull. Mater. Sci. 38, 3 (2015).

5. I.V. Chepkasov, Yu.Ya. Gafner, S.L. Gafner. Journal of Aerosol Science 91 (2016).

6. P. Vogt, P. De Padova, C. Quaresima, et al. Phys. Rev. Lett. 108 (2012).

7. Г.А. Мансури Принципы нанотехнологии. Исследование конденсированных веществ малых систем на молекулярном уровне. М.: Научный мир. 2008. 320c.

8. A.Ye.Galashev, V.A.Polukhin. In Computer Investigation of the Stability and Structure of Si and SiO2 Nanoparticles. Nova Science Publishers. New York. (2012) 130p.

9. M.E. Davila, L. Xian, S. Cahangirov. New J. Phys. 16 (2014).

10. A.E. Galashev, V.A. Polukhin, I.A. Izmodenov et al. Glass. Phys.Chem. 33, 1 (2007).

11. A.E. Galashev, V.A. Polukhin et al. Glass. Phys.Chem. 32 (2006).

12. V.A. Polukhin, N.A. Vatolin. Rasplavy. 3 (1998)

13. V.A. Polukhin, E.A. Kibanova. Russ. J. Phys. Chem. 73, 3 (1999).

14. R. Quhe, Y. Yaun, J. Zheng et al. Sci. Rep. 6 (2012).

15. J. Gao, J. Zhao. Sci. Rep. 2 (2012).

16. В.А. Полухин, Н.С. Митрофанова, Э.Д. Курбанова. Физико-химические аспекты изучения кластеров, наноструктур и наноматериалов, 6 (2014).

17. J.Y. Chen, A. Kutana, C.P. Collier, K.P. Giapis. Science. 310 (2005).

18. V.A. Polukhin, E.D. Kurbanova. J.Phys. Chem. A. 89, 3 (2015).

19. D. Hsieh, D. Qian, L. Wray et al. Nature. 452 (2008).

20. Y. Xu, B.Yan, H.-J. Zhang, J. Wang, G. Xu. Phys. Rev. Letttrs. (FilmsPRL). 111, 10 (2013).

21. V. A. Polukhin, E. D. Kurbanova, A. E. Galashev Russian Metallurgy (Metally). 2012, 8 (2012).

22. V. A. Polukhin, E. D. Kurbanova, A. E. Galashev Russian Metallurgy (Metally). 2014, 8 (2014).

23. Э.Д. Курбанова, В.А. Полухин, Н.С. Митрофанова, Л.К. Ригмант. Физико-химические аспекты изучения кластеров, наноструктур и наноматериалов, 7 (2015). 